Service social

\title{
Dynamique culturelle et service social des groupes. Pratique clinique en milieux multiculturels
}

\section{Kenneth L. Chau}

Volume 39, numéro 1, 1990

Le groupe ici et ailleurs

URI : https://id.erudit.org/iderudit/706454ar

DOI : https://doi.org/10.7202/706454ar

Aller au sommaire du numéro

Éditeur(s)

École de service social de l'Université Laval

ISSN

1708-1734 (numérique)

Découvrir la revue

Citer cet article

Chau, K. L. (1990). Dynamique culturelle et service social des groupes. Pratique clinique en milieux multiculturels. Service social, 39(1), 27-40.

https://doi.org/10.7202/706454ar
Résumé de l'article

Face aux changements majeurs que connaît notre démographie, comment pouvons-nous assurer la pertinence culturelle du service social des groupes auprès des différentes populations auxquelles il s'adresse?

Le présent exposé propose une conception du service social des groupes qui tient compte du sens profond et de la nature unique de la vie dans nos milieux multiculturels. Il traite de questions relatives aux différences culturelles et à l'acculturation en termes d'impacts majeurs sur le bien-être psychologique et social des gens. L'article décrit quatre volets de la pratique du service social des groupes : le traitement et le support; l'éducation et la prévention; la défense des droits de la personne; l'accroissement du pouvoir des individus. Cette approche de l'intervention de groupe constitue une façon de repenser le service social des groupes en fonction de la croissance du multiculturalisme. 
Kenneth L. Chau, professeur, California State University, Long Beach, Californie.

\section{Dynamique culturelle et service social des groupes Pratique clinique en milieux multiculturels ${ }^{1}$}

Kenneth L. Chau

Le présent exposé propose une approche du service social des groupes qui répond au sens profond et à la nature unique de la vie en milieux multiculturels. Cette approche s'appuie sur l'idée que la culture et l'ethnie influencent la nature des problèmes rencontrés par les gens et déterminent les objectifs et les paramètres du service social des groupes.

Notre analyse repose sur notre conviction à l'égard de l'intégrité culturelle et de la force de survie des groupes raciaux et ethniques. Les membres des communautés ethniques ont découvert le sens profond et la nature de la vie en Amérique du Nord, en grande partie à travers des expériences de déplacements et de pertes, de conflits culturels, d'adaptations, de sentiments souvent hostiles à leur endroit et de sentiments d'aliénation sociale associés au fait d'être différents. Puisque l'intervention sociale des groupes devrait tenir compte de ces réalités, il est important de faire ressortir et de comprendre le contexte social et environnemental dans lequel sont plongés les membres des communautés ethniques.

\section{Le contexte : une société multi-ethnique et multiculturelle}

Le contexte auquel nous référons est celui des milieux multiethniques et multiculturels du Canada et des États-Unis. La croissance de la mosaïque ethnique au Canada et celle de la diversité culturelle 
aux États-Unis ont une origine commune. En effet, toutes deux résultent, d'une part, de changements dans la politique de l'immigration, passant d'un système de quotas à un système ouvert (en 1965 aux États-Unis et en 1967 au Canada) et, d'autre part, de l'introduction de politiques libérales telles que le multiculturalisme au Canada en 1971 et, aux États-Unis, le mouvement vers le pluralisme culturel et le respect des droits des citoyens dans les années 1960.

Ces changements ont contribué à la diminution des préjugés racistes dans les milieux politiques et décisionnels et ont favorisé la venue, dans ces deux pays nord-américains, d'immigrants non européens. Aujourd'hui, la mosaïque canadienne est composée de plus de quatre-vingts cultures, et un tiers de la population canadienne n'est d'origine ni britannique ni française. Le recensement de 1981 indique, en effet, que seulement $40 \%$ de la population est d'origine britannique, et $27 \%$, d'origine française. Aux États-Unis, environ un quart de la population n'est pas de race blanche et les projections laissent prévoir une croissance rapide et continue de la population de couleur. Ceci démontre que le Canada et les États-Unis se transforment progressivement en pays multi-ethniques. Dans certains États américains, par exemple la Californie, la population de couleur dépassera bientôt celle de racè blanche. Cette situation existe déjà dans le comté de Los Angeles, région qui connaît la plus grande diversité ethnique en Californie et où la population de couleur atteint $57,9 \%$. Ce contexte est comparable à celui de Toronto, au Canada, où $56 \%$ de la population n'est d'origine ni britannique ni française. Aujourd'hui, la mosaïque culturelle du Canada et des États-Unis nous permet de prendre contact avec la richesse d'une multitude de visions du monde, de systèmes de croyances et de styles de vie. Ainsi, il est inévitable que le Canada et les États-Unis soient fortement influencés par cette "nouvelle majorité ».

Toutefois, la force sociale que représentent la diversité des cultures et l'augmentation de la population ethnique crée de nouveaux défis pour le service social des groupes. Non seulement ces réalités accroissent-elles l'hétérogénéité culturelle des groupes, mais elles nous sensibilisent davantage aux différences de perceptions ou de réponses aux besoins, ainsi qu'à la nécessité de tenir compte de considérations culturelles dans notre travail avec les groupes ethniques.

Idéalement, I'Amérique du Nord aimerait projeter l'image d'une terre d'asile accueillante et hospitalière, où les immigrants s'intègrent graduellement et harmonieusement. Toutefois, la réalité est bien différente, et la vision idéalisée que nous avons de la vie en Amérique du Nord des nouveaux arrivants s'effrite rapidement. Les travailleurs sociaux qui font face aux problèmes de racisme et aux préjugés, qui 
savent ce que signifient l'appartenance à une minorité et l'expérimentation d'une certaine dislocation, ceux-là reconnaîtront que des différences de langue, de comportement social et d'attitudes culturelles peuvent créer des obstacles majeurs au processus d'adaptation des immigrants.

\section{Adaptation culturelle et besoins en santé mentale}

Rien n'est plus révélateur de la nature des obstacles rencontrés par les membres de communautés ethniques dans leur processus d'adaptation que les titres des journaux. En voici quelques exemples tirés du Los Angeles Times :

- "Asian Girls : A Cultural Tug of War" (Lutte culturelle acharnée chez les jeunes filles asiatiques);

- "Clash of Two Worlds Leaves Many in Pain " (Le choc de deux mondes en fait souffrir plusieurs);

- "Family Elders Cling to Old Ways As Young Look to New" (Les aînés s'accrochent aux traditions, les jeunes regardent en avant);

— "Japanese : Young Move On, and the Elderly Are Left Behind"

(Chez les Japonais, les jeunes avancent, les vieux restent en arrière);

— «Racism May Be Making Comeback on California Campuses » (Le racisme semble refaire surface sur les campus californiens).

Telles sont les dures réalités auxquelles ont à faire face les membres de communautés ethniques au fur et à mesure qu'ils s'adaptent à leur nouveau pays, à la culture occidentale, à un mode de vie étranger et, pour certains, à la société urbaine. Assez souvent, ces expériences provoquent une détresse émotive intense. Cet état, que nous avons déjà qualifié de " dissonance socioculturelle", survient lorsqu'une personne vit une incompatibilité entre les attentes et les normes de sa propre culture et celles de sa société d'adoption (Chau, 1989 : 225). Nous décrirons maintenant quatre aspects de l'adaptation culturelle qui produisent cette dissonance socioculturelle, et les illustrerons par des histoires de cas et des articles parus dans les journaux ${ }^{2}$. Nous identifierons plusieurs implications culturelles et proposerons un paradigme de divers volets d'intervention auprès de groupes multiculturels. 


\section{Choc des cultures}

L'éducation des enfants est un sujet qui occasionne très souvent un choc des cultures. Chez nombre de parents asiatiques, la ténacité des croyances et des pratiques culturelles est toujours aussi présente malgré une acculturation apparente, tout particulièrement en ce qui concerne le comportement qu'ils croient " moral » pour leurs enfants et celui qui, selon eux, leur procurera sécurité et prospérité.

"Les règles sont différentes à la maison et à l'école ", disait à ses amis Pearl, une Cambodgienne de 16 ans. "Nous respectons notre père et notre mère comme des dieux. Je n'aurais jamais le cœur de leur désobéir " ajoute-t-elle au cours d'une session de groupe. La jeune fille vit aux États-Unis depuis maintenant douze ans. Elle parle couramment l'anglais, obtient de bonnes notes et espère entreprendre une carrière en sciences politiques et en administration publique. Pearl dit que malgré les taquineries occasionnelles de ses amis, elle accepte facilement l'éducation qu'elle reçoit et même l'éventualité d'épouser un homme choisi par ses parents.

Les enfants inscrits à l'école publique apprennent l'anglais et adoptent le mode de vie américain très rapidement. Toutefois, ils sont coincés entre les attentes et les normes culturelles de deux mondes : d'une part, le monde asiatique qui met l'accent sur l'obéissance et le respect de l'autorité, et d'autre part, un nouveau monde qui encourage l'individualisme et la libre expression. Confrontés à de telles différences culturelles, tous les enfants ne réagissent pas comme Pearl. Plutôt que de répondre aux attentes parentales, nombre d'entre eux se rebellent contre la discipline plus traditionnelle de leurs parents, contre la pression exercée pour qu'ils respectent leurs aînés et leur obéissent et, particulièrement dans le cas des filles, contre les attentes de leurs parents de les voir se comporter selon la tradition.

C'est le cas de Kim pour qui les tentatives de vivre une vie asiatique dans le contexte culturel occidental se sont révélées accablantes. Étudiante en deuxième année à l'université, elle s'est enlevé la vie, incapable de concilier les attentes conflictuelles des deux mondes. Kim était adolescente lorsqu'elle a immigré de Corée et s'est retrouvée plongée dans une culture et un système de valeurs entièrement différents de sa culture d'origine. Elle faisait tout pour être américaine, pour être l'héroïne d'une histoire qui finit bien, pour que ses parents soient fiers d'elle. Elle a lutté pendant des années au cours desquelles tout semblait bien aller, mais finalement, tout s'est écroulé.

Les données recueillies auprès de centres de services sociaux révèlent qu'un nombre croissant de jeunes Asiatiques tentent de se 
suicider, fuguent, abandonnent l'école, se joignent à des " gangs " ou s'adonnent à la drogue ou à la prostitution en réaction au choc des cultures ou aux différences culturelles. Un très fort pourcentage des cas reçus par ces centres ( $40 \%$ à $65 \%$ ) concernent des filles qui ont de graves problèmes à s'ajuster aux attentes de deux cultures différentes.

\section{Pression des parents pour l'atteinte de l'excellence}

Pour nombre de jeunes Asiatiques, les problèmes s'aggravent lorsque leurs parents ont des attentes extrêmement élevées face à leur rendement académique et à leur réussite professionnelle. Plusieurs de ces jeunes ne se voient pas comme les "petits génies » ou les "bolés" qu'une grande partie de la société perçoit.

" II n'y a pas de raison que je ne sois pas aussi populaire que Johnny. "Mike, élève du secondaire, marmonnait à son copain : " J'ai eu de meilleures notes que lui dans presque tous mes cours !" Wayne : "Mon professeur m'a dit qu'un B, c'était bien. Pourquoi maman et papa, eux, trouvent-ils que je ne suis pas assez bon ? Ils m'ont dit que j'étais mauvais et que je devais travailler plus fort pour obtenir un A !"

Plusieurs parents asiatiques n'ont pas pris conscience de la pression énorme qu'ils imposent à leurs enfants, soit en mettant sur leurs épaules leurs propres aspirations et espoirs, qu'ils n'ont pu réaliser eux-mêmes, soit en tentant de les faire correspondre au modèle asiatique. Bien des parents n'ont pas compris que leurs enfants avaient plus besoin d'aide et d'encouragements que de " $A$ ". C'est déjà difficile pour les adolescents de négocier avec leurs parents; lorsqu'ils font face, en plus, à des différences culturelles sur ce qui est reconnu comme bon, correct ou approprié, ils peuvent facilement être submergés.

Ces enfants ont ressenti l'attraction de deux mondes en même temps que l'aliénation de n'appartenir ni à l'un ni à l'autre. Beaucoup de jeunes Asiatiques ont le sentiment d'avoir à se débrouiller tout seuls, et l'ont exprimé au cours de sessions de groupe destinées à les aider à résoudre des problèmes reliés aux attentes parentales et aux difficultés de communication, ou à faciliter leur intégration. Dans ces groupes, ils discutent de différences culturelles et de questions interculturelles, apprennent à interagir avec les gens à l'extérieur du milieu familial ou scolaire, évaluent la signification, pour eux-mêmes et leur famille, des changements de valeurs culturelles. 


\section{Nouvelle dynamique de la communication parent-enfant}

En réaction aux changements de valeurs culturelles et de comportements des enfants, les parents connaissent une hausse de stress et d'aliénation. Ils ont l'impression que leurs enfants leur deviennent étrangers, en raison des attitudes, des manières et des comportements que ceux-ci adoptent. Ils ont le sentiment de perdre la maîtrise de leurs enfants américanisés et, par la même occasion, l'espoir de leur vie, car pour bien des parents asiatiques, les enfants représentent les seuls espoirs et l'unique raison de vivre. Écoutons cette conversation tenue dans un groupe de "rééducation" de parents immigrants et réfugiés, à propos des différences culturelles observées dans les pratiques éducatives, la discipline et les relations familiales :

Maman A : "Mon fils ne reçoit pas l'éducation qu'il devrait recevoir. $\mathrm{Au}$ Japon, les jeunes parlent des bonnes notes qu'ils ont obtenues aux examens. Ici, ils parlent des filles."

Maman B : "Je ne comprends pas pourquoi, mais mon fils a fait un peu de travail pour moi et a demandé à être payé. Dans quelle sorte de monde croit-il être ?"

Maman C: "J'ai dit à ma fille qu'elle devrait rentrer à 11 heures du soir. Vous savez ce qu'elle m'a répondu ? Elle $m^{\prime}$ 'a dit que c'est après 11 heures que le plaisir commence, qu'elle va perdre la face si elle quitte aussi tôt. Est-ce que ne pas perdre la face, c'est plus important que sa propre sécurité ?»

Dans ces circonstances, les attentes des parents se heurtent à celles des pairs qui sont bien différentes. Cela crée beaucoup de stress chez les enfants et provoque ressentiment et mésentente avec leurs parents. Nombre de parents immigrants ne savent pas comment s'en sortir.

Dans d'autres situations, la lutte que doivent mener parents et enfants pour composer avec leur choc culturel respectif amène des problèmes bien différents dans les relations intergénérationnelles. $C^{\prime}$ est le cas de parents qui, ne parlant pas anglais, se sont repliés sur leur communauté ethnique pour rendre l'aliénation plus supportable et, par conséquent, se sont adaptés plus lentement que leurs enfants au mode de vie américain. De leur côté, les enfants doivent avoir des contacts sociaux quotidiens avec un environnement plus étendu et en viennent à s'adapter d'eux-mêmes au contexte culturel. Dans ces familles, le pouvoir est passé des parents aux enfants qui connaissent bien la langue et les subtilités de la société américaine, et qui sont devenus des interprètes et des intermédiaires pour la famille. Ces parents voient leur autorité ébranlée et trouvent souvent difficile de s'adapter à la nouvelle dynamique familiale. Souvent, lorsqu'ils 
réalisent que leur discipline traditionnelle n'obtient plus les résultats escomptés, il est déjà trop tard. En fait, le comportement parental des Asiatiques peut être strict et parfois explosif. L'augmentation des cas de mauvais traitements infligés aux enfants et de violence familiale dans les communautés chinoise, coréenne, vietnamienne et philippine, soulève de sérieuses inquiétudes chez les professionnels de la santé mentale.

\section{Familles en transition}

Les problèmes ne touchent pas que les jeunes, qui peuvent profiter d'une variété d'interventions de groupes. Les problèmes ne sont pas non plus tous reliés à l'aliénation culturelle et aux conflits intergénérationnels. À côté des problèmes associés aux bandes de jeunes et aux tensions raciales sur les campus et leurs environs, et qui font les manchettes, on retrouve de graves difficultés chez plusieurs membres de communautés ethniques et chez de nouveaux arrivants aux prises avec une détresse émotive dans laquelle les ont plongés le chômage, les emplois subalternes, les difficultés de langue, la solitude, l'isolement et les ajustements, situations qui sont le lot des "familles en transition".

Selon le "Asian Pacific Family Center" de Rosemead, en Californie, la grande majorité (67\%) des Asiatiques qui ont besoin de soins psychologiques et psychiatriques sont d'âge moyen. Imaginons par exemple le sort d'un médecin qui devient technicien de laboratoire, d'un enseignant du secondaire qui devient concierge, ou celui de femmes qui, n'ayant jamais été sur le marché du travail, trouvent un emploi alors que leurs maris n'y parviennent pas. De nombreux hommes asiatiques ont été incapables de surmonter le choc culturel et la perte de leur estime de soi. Dans bien des cas, les hommes ont plus de difficulté à trouver du travail que les femmes ou alors, parce qu'ils ne connaissent pas bien l'anglais ou que leur formation n'est pas reconnue, ils ne peuvent avoir accès à des postes comparables à ceux qu'ils occupaient auparavant; ils se sentent diminués d'avoir à travailler comme concierge ou comme technicien de laboratoire. Les femmes, que les besoins financiers ont amenées sur le marché du travail, peuvent occuper des postes subalternes sans connaître une telle détresse psychologique.

Les femmes ne rapportent pas que des chèques de paie à la maison. Elles y ramènent aussi de nouvelles attitudes, s'affirment de plus en plus au sein de la famille et exigent une plus grande égalité, ce qui affaiblit encore davantage l'image que les maris ont d'eux-mêmes. Les changements d'attitudes et de rôles chez les femmes créent une nouvelle dynamique entre hommes et femmes, ce qui provoque des 
déchirements dans la vie familiale traditionnelle, principalement en ce qui concerne les rôles sexuels et toutes les autres formes de hiérarchie sociale.

La majorité des Asiatiques qui ont besoin de soins psychologiques et psychiatriques sont des hommes emprisonnés dans des rôles de dépendance ou dans des rôles qu'ils ne désirent pas, ainsi que des femmes qui tolèrent mal les tensions émotives reliées aux multiples changements de rôles. II y a aussi un assez grand nombre d'Asiatiques plus âgés qui font face à des problèmes de solitude, d'isolement, ou qui perdent leurs réseaux de soutien. Tout ceci a pour effet de faire grimper, chez les familles des communautés culturelles, l'incidence des maladies affectives telle la dépression, ainsi que l'abus de drogues, la violence familiale et la détérioration des relations conjugales.

II n'est toutefois pas facile pour les Asiatiques d'avoir recours à une aide professionnelle lorsque le stress et la douleur émotive dégénèrent en problèmes psychologiques et psychiatriques. II existe une terrible stigmatisation culturelle dans la communauté asiatique en ce qui a trait à la maladie mentale. Une réticence culturelle peut aussi empêcher nombre de clients potentiels des services de santé mentale de recourir à l'aide et aux conseils professionnels dont ils ont besoin.

Où se situe le service social des groupes dans ce contexte ethnique ? Comment pourrait-il être culturellement sensible à de telles situations critiques? De quelles façons le service social des groupes pourrait-il "mener la marche, et ne pas suivre le convoi " (Carey-Bélanger, 1989) dans sa manière de répondre aux problèmes d'incompatibilité culturelle et aux besoins d'adaptation des membres des communautés ethniques? Quels éléments culturels devraient être pris en considération lorsque nous abordons les questions d'intervention clinique, de rôles du praticien et de distribution des services?

\section{Considérations culturelles en service social des groupes}

Premièrement, il est essentiel d'avoir une vision étendue du service social des groupes. Tous les problèmes de différences culturelles et d'adaptation ne pourront pas être solutionnés efficacement par une approche limitée de traitement ou par une approche non directive. Par exemple, la thérapie de groupe basée sur le développement de l'insight, l'évolution personnelle et l'adaptation 
psychologique du moi ne peut être utile que pour un nombre limité de problèmes (Tsui et Schultz, 1985).

D'autres formes d'intervention de groupe peuvent s'avérer d'une très grande pertinence pour répondre aux multiples facettes des problèmes d'ajustement, si elles adoptent une perspective préventive, développementale ou éducative, ou si elles visent la défense des droits ou l'accroissement du pouvoir de l'individu. Soutenus par un ensemble intégré de services à la famille, ces modes d'intervention de groupe peuvent contribuer à renforcer l'intégrité des familles ethniques. Plusieurs formes de groupes, par exemple les groupes à court terme, à durée limitée, et les groupes de tâche (Garvin, Reid et Epstein, 1976), correspondent aux attentes des Asiatiques qui aiment être dirigés et orientés vers des solutions pratiques. Les groupes structurés (Middleman, 1980; Papell et Rothman, 1980), centrés sur un sujet bien défini et dont le plan d'action est concret, seraient particulièrement attrayants et utiles pour atteindre des objectifs d'apprentissage social et interpersonnel.

Deuxièmement, le service social auprès de groupes multiculturels doit mettre l'accent à la fois sur le changement individuel et sur le changement institutionnel. L'utilisation des processus de groupe pour aider les gens à mieux s'adapter et à devenir compétents doit tendre vers l'élaboration de politiques et de procédures organisationnelles culturellement appropriées, de façon à faciliter l'accessibilité et l'utilisation du plus grand nombre de services possibles. Tout en aidant leurs clients à travailler en groupe, à en utiliser le programme, à prendre des décisions de groupe, etc., les praticiens appliquent des macrostratégies pour remettre en cause des pratiques institutionnalisées qui compromettent l'équité, la justice ou le droit d'accès des clients.

Troisièmement, l'interdépendance et l'aide mutuelle peuvent être des concepts puissants en intervention de groupe auprès de clientèles multiculturelles. Les praticiens ne se contentent pas de fournir des services et d'aider la clientèle ethnique à fonctionner plus efficacement dans son nouvel environnement, mais cherchent aussi à lui « enseigner » ou à la soutenir dans ses propres efforts d'organisation, que ce soit en termes d'entraide, d'aide mutuelle ou de défense de ses droits. Ceci peut finalement aider les membres des communautés culturelles à acquérir du pouvoir et à l'exercer eux-mêmes, à développer ou à mobiliser des ressources et à contester ou changer leur réalité. Ces interventions augmentent l'efficacité personnelle et la solidarité du groupe, et encouragent les membres à prendre la maîtrise des événements de leur vie malgré le déracinement culturel et les multiples pertes qu'ils ont subis.

Quatrièmement, l'intervention auprès de groupes multiculturels doit mettre en valeur la morale du pluralisme culturel, ceci en 
acceptant les différences culturelles et en respectant les forces inhérentes à ces différences. Au cours de leur processus d'intégration à la société américaine, les immigrants ont besoin d'en arriver à un accord avec eux-mêmes, avec leur propre identité. Comme la culture et l'ethnie jouent un rôle majeur dans la formation du concept de soi ainsi que dans le développement des habiletés cognitives et des aptitudes à se débrouiller (Sotomayor, 1977), les immigrants ont besoin d'aide pour protéger, maintenir et valoriser leur propre identité culturelle, qui est à la base de l'estime de soi et du bien-être de l'individu. En encourageant les gens à être fiers de leur héritage culturel tout en les incitant à adopter un comportement adapté à la culture de leur pays d'adoption et à celle des autres groupes ethniques, nous pouvons attirer leur attention sur des points de convergence et de divergence entre les cultures et ainsi, favoriser leur compatibilité culturelle.

Faisant référence à l'intervention auprès des immigrants et des réfugiés, Evelyn Lee, directrice des programmes au Département de psychiatrie du San Francisco General Hospital, disait : "Notre but n'est pas de fabriquer plus de maïs soufflé, ces petits grains jaunes qui, sous la pression, deviennent blancs » (1989). En service social des groupes auprès d'une clientèle multiculturelle, il n'est pas nécessaire, pour aider les gens à s'adapter à la culture américaine, de les pousser à abandonner leur propre identité culturelle et à devenir " blancs ". L'adaptation se fera aussi bien, sinon mieux, si nous les aidons à reconnaître les frontières entre les cultures et à intégrer les éléments qui leur conviennent. Cette façon de voir est compatible avec la conception de Green (1982) du rôle de "médiateur de frontières" qu'il attribue au travailleur social. Dans ce rôle, l'intervenant a pour objectif d'aider les membres des communautés ethniques à atteindre, au cours de leur processus d'adaptation, un équilibre entre la conservation de leur culture et l'accommodation à la culture dominante et à celle d'autres groupes ethniques.

\section{Quatre volets caractéristiques du service social des groupes en milieu multiculturel}

Compte tenu des considérations culturelles mentionnées plus tôt, nous proposons une approche du service social des groupes dont la procédure, les stratégies et les programmes ont pour objectifs quatre volets majeurs qui ont un impact sur le bien-être des membres des communautés ethniques (Chau, 1989). En bref, il s'agit des volets d'intervention suivants : I'adaptation culturelle, la conscientisation à l'intérieur du groupe, les relations entre les groupes et les droits des minorités. 


\section{Adaptation culturelle}

Ici, les groupes ont pour but de rehausser la compétence personnelle de façon à faire face à l'incompatibilité culturelle ou à augmenter la compatibilité culturelle.

L'intervention de groupe planifiée vise à développer de nouvelles habiletés, de nouvelles ressources et de nouveaux réseaux de soutien, tout en renforçant ou en appuyant les éléments positifs des pratiques culturelles et des stratégies d'adaptation de chacun. Dans l'optique où la connaissance et l'information contribuent à éliminer la peur de l'inconnu et à améliorer la capacité personnelle d'adaptation aux différences culturelles, les groupes visent à familiariser les gens à leur nouvel environnement, à les orienter vers les ressources de leur milieu d'adoption et à les préparer aux nouvelles attentes culturelles, et ainsi à faciliter leur adaptation à de nouveaux environnements. D'autres groupes orientés vers la résolution de problèmes se penchent sur des besoins de nature personnelle ou sur la discussion de questions interculturelles, alors que des groupes de croissance recherchent un enrichissement personnel et social. Dans ce type d'intervention, il s'agit d'adopter une stratégie normative qui vise à socialiser la personne, à l'entraîner ou à l'éduquer à rencontrer les attentes et à se comporter selon les normes de sa société d'adoption, tout en prêtant attention à ses soubresauts culturels et à ses demandes d'aide.

\section{Conscientisation à l'intérieur du groupe}

Ici, l'intervention de groupe a pour but de stimuler chez le participant la conscience des valeurs ethniques, et d'asseoir sur de nouvelles bases son pouvoir et ses ressources, pour ainsi faire face aux problèmes de cohésion créés par la perte du soutien et des liens familiaux, et pour contrer l'impuissance politique et sociale des communautés ethniques. Les stratégies utilisées au cours du processus d'intervention visent à redonner du pouvoir (Hirayama et Hirayama, 1986; Pernell, 1986). Plusieurs types de groupes sont particulièrement efficaces pour favoriser une identité positive et une force de groupe. Ces groupes encouragent chez les participants la fierté d'appartenir à leur communauté ethnique, développent le sens du leadership à l'intérieur de la communauté, mettent sur pied des organisations et favorisent l'interdépendance et l'aide mutuelle par la création de réseaux et de coalitions. Ces groupes permettent de mobiliser la vitalité et la force latente des membres des communautés ethniques et de développer des ressources au sein de leur communauté pour donner aux gens un sentiment de pouvoir et de contrôle accrus sur leurs réalités quotidiennes. 


\section{Relations entre les groupes}

L'intervention de groupe peut avoir pour cible les préjugés raciaux, la tension et l'hostilité associés à l'insensibilité culturelle et aux stéréotypes véhiculés entre les groupes dominants et les groupes ethniques, et entre les groupes raciaux et ethniques eux-mêmes. Souvent, l'insensibilité culturelle et la xénophobie sont le fruit d'un manque d'information et de compréhension, ou encore de la distorsion des attitudes ou des comportements que peuvent avoir les différents groupes culturels les uns par rapport aux autres et par rapport aux différences culturelles.

Ici, l'intervention de groupe ne doit pas seulement adopter une stratégie rééducative, c'est-à-dire offrir des groupes de dialogue, de conscientisation culturelle, ou des groupes multiculturels axés sur la compréhension interraciale. Elle doit en outre encourager les contacts et la collaboration entre les groupes comme moyens de favoriser la compréhension, l'acceptation et l'intégration interraciales.

\section{Droits des minorités}

Les institutions sociales sont fortement conditionnées par la culture; elles répondent en priorité aux besoins des groupes dominants et négligent de fournir des services équitables aux autres groupes. L'intervention de groupe auprès de clientèles multiculturelles comprend donc un autre volet, celui de la défense des droits, qui a pour objectif de promouvoir l'équité et la sensibilité culturelle dans les politiques, procédures et services institutionnels.

Parmi les groupes de santé mentale et de services sociaux qui mettent l'accent sur ce volet d'intervention, on retrouve entre autres les regroupements pour la défense des droits des bénéficiaires, les comités de bénéficiaires en milieu hospitalier, et d'autres groupes de prise en charge ou d'entraide œuvrant au sein d'un organisme. Ces groupes aident les clients à obtenir plus de contrôle sur les pratiques institutionnelles et à s'assurer que les services qu'ils reçoivent répondent mieux à leurs besoins et leur sont plus facilement disponibles et accessibles.

\section{Conclusion}

Ces quatre volets de l'intervention fournissent donc un cadre général pour le service social des groupes en milieu multiculturel, cadre qui embrasse à la fois des pespectives de traitement et de développement, d'éducation, de prévention et d'accroissement du pouvoir des membres. 
Cette conception du service social des groupes constitue une formule intéressante pour aborder les questions d'incompatibilité culturelle, ainsi que les différents aspects de l'adaptation qui ont un impact important sur le bien-être des membres des communautés culturelles vivant dans des milieux multi-ethniques et multiculturels. Elle propose une façon de repenser le service social des groupes (Breton, 1989) en utilisant les forces de notre tradition et en $y$ apportant des innovations pour relever les défis posés par nos milieux de plus en plus multiculturels.

Nous espérons que cet article aura su mettre en relief l'idée que la sensibilité culturelle est une condition sine qua non pour intervenir efficacement auprès des membres de communautés culturelles et répondre adéquatement à leurs besoins. II est maintenant essentiel de développer un contexte et une approche du service social des groupes qui répondent aux réalités culturelles des groupes ethniques. Si la conception de l'intervention de groupe décrite ici nous incite à continuer à travailler pour rendre le service social des groupes plus viable en milieu multiculturel, l'objectif de cet article aura alors été atteint.

\section{Notes}

1 Texte traduit par Lyne Champoux. Une grande partie du présent article est tirée d'une conférence présentée au symposium de Montréal, et dont le contenu sera aussi publié dans Groupwork, en Angleterre, sous le titre : "Social Work with Groups in Multicultural Contexts". Les éditeurs de Groupwork en ont autorisé l'utilisation.

${ }^{2}$ Quelques-uns des exemples utilisés pour illustrer différents aspects de l'adaptation culturelle sont des versions adaptées d'histoires de cas ou de récits parus dans des journaux locaux, particulièrement le Los Angeles Times. II s'agit, entre autres, des articles suivants : Vicki Smith, "Male Asian Immigrants Suffering Cultural Shock», San Gabriel Valley Tribune, 19 septembre 1988; la série de Mark Arax, "The Asian Impact ", Los Angeles Times, avril 1987; David Haldane, "Immigrant Ills. Asian Girls : A Cultural Tug of War ", Los Angeles Times, 24 septembre 1988; Lennie La Guire et Shawn Hubler, "Asian Pacific Sketchbook», Los Angeles Herald Examiner, 6-27 mai 1988.

\section{Références}

BREton, Margot (1990). "Leçons à tirer de nos traditions en service social des groupes", Service social, vol. 39, n' $1:$ 13-26.

CAREY-BÉlANGER, Élaine (1989). "Influence des changements dans le contexte socio-économico-politique sur la pratique du service social des groupes : le cas du Québec ", communication présentée à la séance d'ouverture, 
$11^{\mathrm{e}}$ symposium annuel de l'Association pour l'avancement du service social des groupes, tenu en octobre 1989, à Montréal.

CHAU, Kenneth L. (1989). "Sociocultural dissonance among ethnic minority populations ", Social Casework, vol. 70, n 4 : 224-230.

Garvin, Charles D., W. ReID et L. EPSTEIN (1976). "A task-centered approach », dans Robert W. Roberts et Helen Northen (éd.), Theories of social work with groups. New York: Columbia University Press.

GREEN, James W. (1982). Cultural Awareness in the Human Services. Englewood Cliff, N.J. : Prentice-Hall.

HiRayama, Hisashi et Kasumi HiRayama (1986). "Empowerment through group participation : Process and goal », dans Marvin Parnes (éd.), Innovations in Social Group Work: Feedback from Practice to Theory. New York : Haworth Press.

LEE, Evelyn, citée dans John KRICK (1989). "Culture crash», Mother Jones. (October).

MIDDLEMAN, Ruth R. (1980). "The pursuit of competence through involvement in structured groups ", dans A.M. Maluccio (éd.), Building Competence in Clients. New York : Free Press.

PAPELL, C. et B. RothMAN (1980). "Relating the mainstream model of social work with groups to group psychotherapy and the structured group approach ", Social Work With Groups, vol. 3, $\mathrm{n}^{\circ} 2$ : 5-23.

PernelL, Ruby B. (1986). "Empowerment and social group work", dans Marvin Parnes (éd.), Innovations in Social Group Work: Feedback from Practice to Theory. New York : Haworth Press.

SOTOMAYOR, Marta (1977). "Language, culture and ethnicity in developing selfconcept », Social Casework, vol. 58 (April).

TSUI, P. et G. SChULTZ (1985). "Failure of rapport: Why psychotherapeutic engagement fails in the treatment of Asian clients ", American Journal of Orthopsychiatry, vol. 55. 